\title{
Pneumonia: Tratamento e Evolução
}

\section{Pneumonia: Treatment and Evolution}

\author{
Daniel Machado ${ }^{1}$ \\ Leandro Tristão Abi-Ramia de Moraes ${ }^{1}$ \\ Mariana de Oliveira Escareli ${ }^{1}$ \\ Itamar Alves Vianna ${ }^{2}$
}

Artigo

Original

Original

Paper

Palavras-chave:

Pneumonia

Antimicrobianos

Agentes Etiológicos

\section{Resumo}

Tendo em vista o uso indiscriminado dos antimicrobianos atualmente, este trabalho, através de um relato de caso e de uma pesquisa bibliográfica, discute sobre como aplicar os antibióticos empiricamente. Uma vez que o desconhecimento do agente etiológico, assim como da sua sensibilidade aos antimicrobianos pode resultar no insucesso da terapêutica e aumentar a resistência do microorganismo aos antibióticos. Nós relatamos o tratamento de uma criança de um ano e dez meses de idade, hospitalizada com pneumonia, sendo que o projeto da pesquisa foi aprovada pelo CoEPS sob o protocolo 213/09 em 14/12/2009.

\begin{abstract}
This work, through a case report and a literature review, discusses how to apply antibiotics empirically, once that nowadays the use of antimicrobial is indiscriminate. The ignorance of the agent, as well as its sensitivity to antimicrobials can result in the failure of the therapy and it can increase the microorganisms resistance to the antibiotics. We report the treatment of a child aged one year and ten months old, hospitalized because of pneumonia. The project of the research was approved by COEPS under the register 213/09 in 14/12/2009.
\end{abstract}

Key words:

Pneumonia

Antimicrobial

Agents

Etiologic Agents 


\section{Introdução}

“As infecções respiratórias agudas são responsáveis por um terço das mortes e metade das hospitalizações e consultas médicas entre menores de cinco anos nos países em desenvolvimento." (CÉSAR, 1997, p. 54).

Para KLIEGMAN et al (2006, p. 507), a pneumonia é uma infecção do trato respiratório inferior, que compromete as vias aéreas e o parênquima, com ou sem consolidação dos espaços alveolares. Quando nos referimos à pneumonia lobar, fazemos entender uma pneumonia "típica", localizada em um ou mais lobos do pulmão, em que o lobo ou lobos afetados estão completamente consolidados. Para pneumonia "atípica" cabem outros padrões que diferem da pneumonia lobar. O termo broncopneumonia refere-se à inflamação do pulmão, que é centrada nos bronquíolos e leva à produção de um exsudato mucopurulento, que obstrui algumas dessas pequenas vias aéreas e causa consolidações distribuídas em focos nos lóbulos adjacentes. A pneumonia aguda, assim como qualquer doença aguda, tem um curso acelerado, com convalescença ou morte no período de três meses.

Cerca de $10 \%$ a $20 \%$ de todas as crianças menores de cinco anos, nos países pobres, apresentam pneumonia aguda (PA) a cada ano (FERREIRA e BRITO, 2003, p. 478). No Brasil, a pneumonia é a terceira causa da mortalidade infantil (FERNANDES , 2004, p. 208), em $1998,5,4 \%$ e $12,8 \%$ dos falecimentos de menores de um ano e de crianças entre um e quatro anos, respectivamente, foram produzidos pela PA, em 1999, 8,1\% das hospitalizações em todas as idades foram produzidas por PA (FERREIRA e BRITO, 2003, p. 478). No município de Volta Redonda, em 2005, houve duas mortes por pneumonia em crianças entre um e quatro anos de idade (MINISTÉRIO DA SAÚDE, 2005) ${ }^{1}$.

Pneumonia aguda é causa de morte evitável por medidas simples, acessíveis, de baixo custo e que não exigem tecnologia diagnóstica nem recursos terapêuticos sofisticados, na maioria das vezes (idem FERREIRA e BRITO).

\section{Relato de Caso}

Paciente do sexo masculino, com 1 ano e 10 meses de idade, deu entrada no hospital com queixa principal de febre. Na história da moléstia atual, há relato de febre há 7 dias, de $38 / 39^{\circ} \mathrm{C}$, acompanhada de tosse e queda do estado geral. História de asma em uso de Predsim ${ }^{\circledR}+$ nebulização (fenoterol e brometo de ipratrópio). No exame físico, o paciente encontrava-se prostrado, hipoativo, dispneico, com a frequência respiratória (FR) de 46 ipm e retração de fúrcula esternal. A avaliação do aparelho respiratório mostrava murmúrio vesicular (MV) audível universalmente, apresentando sibilos e roncos difusos. A temperatura axilar (TAx.) encontrada no momento do exame foi de $39,5^{\circ} \mathrm{C}$.

Os resultados de relevância encontrados nos exames solicitados com o paciente internado foram: bastões $5 \%$, VHS: $100 \mathrm{~mm}$, plaquetas: $543.000 / \mathrm{mm} 3$. Foi iniciada terapêutica com penicilina cristalina (200.000 UI/ kg/ dia em quatro doses), nebulização com fenoterol e brometo de ipratrópio, hidratação venosa $(\mathrm{HV})$ e hidrocortisona $(5 \mathrm{mg} / \mathrm{kg} /$ dose a cada seis horas).

No segundo dia de internação, a TAx. era de $36^{\circ} \mathrm{C}$ e a FR de $32 \mathrm{ipm}$, sem sinais de esforço respiratório. A ausculta respiratória mostrava sibilos difusos. A prescrição foi mantida com suspensão do brometo de ipratrópio. Ao terceiro dia, a FR foi de 54 ipm. O MV encontrava-se diminuído, associado a crepitações e roncos difusos. A conduta foi mantida.

Na avaliação do quarto dia de internação, o paciente apresentava FR de 48 ipm, leve tiragem subcostal, além de MV diminuído com roncos e crepitações difusas. Foi identificada piora da imagem radiológica e do estado geral. À prescrição, foi acrescentado oxigênio sob cateter, trocada a penicilina cristalina por oxacilina (200mg/ kg/ dia em quatro doses) mais ceftriaxone (100 mg/ kg/ dia em duas doses) e suspensa a hidrocortisona.

Ao avaliar o aparelho respiratório no quinto dia de internação, foi evidenciado FR: $40 \mathrm{ipm}$, roncos difusos, estertores em pulmão direito e sopro tubário em pulmão esquerdo. 
A TAx. aferida foi de $36,5^{\circ} \mathrm{C}$. A conduta foi alterada apenas na redução da HV.

No dia seguinte, o paciente apresentavase afebril, ativo e reativo. Foram evidenciados, além do esforço respiratório, MV diminuído, estertores e roncos difusos. A conduta, nesse dia, ficou inalterada. No sétimo dia de internação o paciente apresentava melhora do estado geral com diminuição da tosse, ainda produtiva, e dos estertores e roncos, a FR foi de 28 ipm e a TAx. de $36,5^{\circ} \mathrm{C}$. Foi solicitada radiografia de tórax e a conduta medicamentosa foi mantida.

Nas avaliações do oitavo e nono dias de internação, houve melhora do estado geral com MV presente, estertores difusos e roncos esparsos, ausência de febre. A prescrição foi alterada com suspensão da HV. Ao décimo dia o lactente apresentava bom estado geral, taquipneico, FR: $32 \mathrm{ipm}$, com estertores em ambos os pulmões e sua TAx. era de $36^{\circ} \mathrm{C}$. Foi solicitado novo raio-x de tórax e a conduta medicamentosa foi mantida.

No $11^{\circ}$ dia de internação, o paciente estava em bom estado geral, mas, ainda, apresentando estertores, principalmente em pulmão esquerdo. A TAx. era de $36,5^{\circ} \mathrm{C}$. A conduta foi alta hospitalar com receita de cefalexina (275mg/ dose, quatro vezes ao dia, por sete dias) e dipirona (oito gotas em caso de febre, até quatro vezes ao dia).

A perfusão encontrou-se sem alterações durante toda a internação.

\section{Discussão}

Para as crianças com idade igual ou maior que 2 meses, a classificação em pneumonia grave baseia-se na presença de tiragem subcostal. A classificação em pneumonia muito grave baseia-se na presença de convulsões, sonolência, estridor em repouso, desnutrição grave, ausência da ingestão de líquidos ou sinais de insuficiência respiratória grave, como cianose central (NASCIMENTOCARVALHO e SOUZA-MARQUES, 2004, p. 383 apud AIDPI, 2000, p. 38). Destaca-se a importância da contagem da frequência respiratória da criança e a pesquisa de tiragem subcostal antes de qualquer manobra para a realização do exame físico, uma vez que o recomendado é que o paciente esteja tranquilo ou até mesmo dormindo (AMARAL e PAIXÃO, 2004, p. 24).

Devido à dificuldade do pronto diagnóstico etiológico das pneumonias, o tratamento instituído por via de regra é empírico ${ }^{2}$.

Tabela 1. Agentes etiológicos mais prováveis de pneumonia comunitária entre 4 meses e 4 anos de idade (NASCIMENTO-CARVALHO e SOUZA-MARQUES, 2004, p. 383) ${ }^{3}$ :

\begin{tabular}{|l|l|}
\hline \multicolumn{1}{|c|}{ AGENTE } & \multicolumn{1}{c|}{ ASPECTO CLÍNICO RELEVANTE } \\
\hline $\begin{array}{l}\text { Vírus sincicial respiratório, parainfluenza, influenza, } \\
\text { adenovírus, rinovírus. }\end{array}$ & $\begin{array}{l}\text { Frequentemente causam pneumonia entre as crianças mais } \\
\text { jovens deste grupo etário. }\end{array}$ \\
\hline Streptococcus pneumoniae. & $\begin{array}{l}\text { Causa mais provável de pneumonia lobar ou segmentar, } \\
\text { mas também pode causar outras formas }\end{array}$ \\
\hline Haemophilus influenzae. & $\begin{array}{l}\text { Tipo b em desaparecimento devido ao uso da vacina } \\
\text { conjugada em larga escala; outros tipos e não-tipáveis } \\
\text { também causam pneumonia. }\end{array}$ \\
\hline Staphylococcus aureus. & $\begin{array}{l}\text { Doença grave, freqüentemente complicada entre os mais } \\
\text { jovens deste grupo etário. }\end{array}$ \\
\hline Mycoplasma pneumoniae. & $\begin{array}{l}\text { Exposição a paciente bacilífero, ausência de resposta ao } \\
\text { tratamento para os agentes mais comuns. }\end{array}$ \\
\hline Mycobacterium tuberculosis. & Crianças mais velhas neste grupo etário. \\
\hline
\end{tabular}

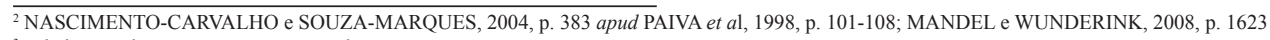


Para os indivíduos com mais de 2 meses de idade e que necessitam de tratamento hospitalar, o esquema antimicrobiano deverá ser escolhido conforme a gravidade, por exemplo: para os casos graves, inicia-se a penicilina cristalina ou a ampicilina, e para os casos muito graves, a oxacilina associada ao cloranfenicol ou à ceftriaxona. ${ }^{4}$

Tabela 2. Posologia dos principais antimicrobianos para tratamento de pneumonia em crianças com idade igual ou maior do que 2 meses de idade e adolescentes (NASCIMENTOCARVALHO e SOUZA-MARQUES, 2004, p. 385). ${ }^{5}$

\begin{tabular}{|l|l|l|l|}
\hline $\begin{array}{c}\text { TRATAMENTO HOSPITALAR } \\
\text { (VIA ENDOVENOSA) }\end{array}$ & \multicolumn{1}{|c|}{ DOSAGEM } & $\begin{array}{c}\text { INTERVALO ENTRE } \\
\text { TOMADAS (HORAS) }\end{array}$ & $\begin{array}{c}\text { DURAÇÃO } \\
\text { (DIAS) }\end{array}$ \\
\hline Penicilina cristalina & $200.000 \mathrm{UI} / \mathrm{kg} / \mathrm{dia}$ & $6 \mathrm{em} 6$ & $7 \mathrm{a} 10$ \\
\hline Ampicilina & $150 \mathrm{mg} / \mathrm{kg} / \mathrm{dia}$ & $6 \mathrm{em} 6$ & $7 \mathrm{a} 10$ \\
\hline Oxacilina & $200 \mathrm{mg} / \mathrm{kg} / \mathrm{dia}$ & $6 \mathrm{em} 6$ & $7 \mathrm{a} 10$ \\
\hline Cloranfenicol & $50 \mathrm{mg} / \mathrm{kg} / \mathrm{dia}$ & $6 \mathrm{em} 6$ & $7 \mathrm{a} 10$ \\
\hline Ceftriaxona & $75 \mathrm{mg} / \mathrm{kg} / \mathrm{dia}$ & $24 \mathrm{em} 24$ & 21 \\
\hline Vancomicina & $40 \mathrm{mg} / \mathrm{kg} / \mathrm{dia}$ & $6 \mathrm{em} 6$ & \\
\hline
\end{tabular}

Assim foi conduzido o paciente do caso, sendo utilizada a penicilina cristalina $(200.000$ $\mathrm{UI} / \mathrm{kg} / \mathrm{dia}$, divididas em quatro doses) como o antimicrobiano de escolha ao se detectar um quadro de pneumonia grave. Após 48h, o quadro evoluiu para uma pneumonia muito grave, havendo, então, uma adequação do tratamento empírico substituindo-se a penicilina cristalina por oxacilina $(200 \mathrm{mg} / \mathrm{kg} / \mathrm{dia}$ em quatro doses) mais ceftriaxone $(100 \mathrm{mg} / \mathrm{kg} / \mathrm{dia}$ em duas doses).

Por outro lado, quando há possibilidade da investigação e detecção do agente etio- lógico causador da pneumonia em questão, usam-se antibióticos apontados através do antibiograma, sendo estes, mais eficazes para cada patógeno.

Desse modo, para S. aureus recomendase oxacilina, cefalosporinas de primeira geração, ou amoxacilina com ácido clavulânico. Em casos resistentes são utilizados vancomicina, teicoplamina, aminoglicosídeo + vancomicina, associações de oxacilina ou vancomicina à rifampicina ou aminoglicosídeo (NASCIMENTO-CARVALHO e SOUZAMARQUES, 2004, p. 384).

Tabela 3. Terapia antimicrobiana para pneumonia causada por patógenos específicos (KLIEGMEN et al, 2006, p. 513): ${ }^{6}$

\begin{tabular}{|l|l|l|}
\hline \multicolumn{1}{|c|}{ PATÓGENO } & \multicolumn{1}{|c|}{ RECOMENDADO } & \multicolumn{1}{c|}{ ALTERNATIVO } \\
\hline S. pneumoniae & $\begin{array}{l}\text { Ceftriaxona, cefotaxima, penicilina G } \\
\text { ou penicilina V }\end{array}$ & $\begin{array}{l}\text { Cefuroxima axetila, eritromicina ou } \\
\text { vancomicina }\end{array}$ \\
\hline S. aureus & Nafcilina ou oxacilina & Vancomicina \\
\hline
\end{tabular}

Mesmo sugerindo os antibióticos específicos para cada agente etiológico, deve-se seguir o estudo epidemiológico local (KLIEGMEN et al, 2006, p. 513).

Segundo MANDEL e WUNDERINK (2008, p. 1623-1624), pacientes que apresen- tam necessidade de um tratamento hospitalar da pneumonia adquirida na comunidade devem ser submetidos a uma radiografia de tórax, a uma avaliação microbiológica imediatas e receber antibióticoterapia segundo a coloração de Gram do escarro e o conhecimento da sensibilidade atual

\footnotetext{
${ }^{4}$ NASCIMENTO-CARVALHO e SOUZA-MARQUES, 2004, p. 384; KLIEGMEN et al, 2006, p. 512-513.

${ }^{5}$ Tabela completa encontra-se no texto do autor

${ }^{6}$ Tabela completa encontra-se no texto do autor.
} 
dos antimicrobianos. A penicilina ou a ampicilina são os fármacos de escolha para infecções por pneumococos. Cepas resistentes à penicilina são sensíveis a vancomicina, e a terapia deve incluir esse antibiótico. Se não estiver disponível o resultado da coloração de Gram, recomenda-se fazer tratamento empírico de pacientes hospitalizados com um $\beta$-lactâmico sozinho ou combinado com um inibidor de $\beta$-lactamase.

Medidas de apoio devem ser adotadas, tais como: oxigênio suplementar e líquidos intravenosos; auxilio da eliminação de secreções e assistência ventilatória serão empregados se necessário (MANDEL e WUNDRINK, 2008, p. 1624). Essas medidas também foram somadas aos antibióticos no tratamento da criança do caso em questão, a fim de oferecer suporte e auxiliar na sua recuperação. Havendo melhora do estado clínico do paciente, o tratamento pode ser mudado de intravenoso para oral dentro de 3 dias, a fim de completar um ciclo de 7 a 10 dias sem interromper o tratamento (MANDEL e WUNDRINK, 2008, p. 1627), para o paciente com a pneumonia relatada, foi receitado, na alta hospitalar, cefalexina oral, após as remissões dos sintomas e da gravidade clínica.

Dessa maneira, quando o tratamento da pneumonia for empírico e nosocomial, como primeira escolha, deve-se utilizar penicilina cristalina ou ampicilina. No caso da detecção de gravidade, usa-se oxacilina associada a cloranfenicol ou a ceftriaxona, além da associação de um inibidor da $\beta$-lactamase, como por exemplo, amoxicilina com ácido clavulânico ou ampicilina com sulbactan na suspeita de Haemophilus influenzae ou outro agente produtor de $\beta$-lactamase.

\section{Referências}

1. CÉSAR, Juraci A. et al. Hospitalização por pneumonia: influência de fatores socioeconômicos e gestacionais em uma coorte de crianças no Sul do Brasil. São Paulo. Rev. Saúde Pública v. 31, n. 1. p. 53-61. fev. 1997, Disponivel em: <http:// www.scielo.br/scielo.php?pid=S0034$89101997000100008 \& \quad$ script $=$ sciarttext\& tlng=es $>$. Acessado em: 09. out. 2009.
2. KLIEGMAN, Robert M. et al. Pneumonia. In: Nelson - Princípios de Pediatria. Tradução da 5.ed. Rio de Janeiro: Elsevier, 2006. cap. 110, p. 507-514.

3. FERREIRA, Otelo S. e BRITO, Murilo C. A. Pneumonia aguda-tema que todos devemos estudar. Porto Alegre. Jornal de Pediatria.v. 79, n. 6.p.478-479.nov./dez.2003. Disponível em: $<$ http://www.scielo.br/scielo.php?pid=S0021$75572003000600003 \& \mathrm{script}=\mathrm{sci}$ arttext\&tlng=es>. Acessado em: 09.out.2009.

4. FERNANDES, José C.; FERNANDES, Iracema C. O. F. e EJZENBERG, Bernardo. Pneumonias Bacterianas. In: MARCONDES, Eduardo et al. Pediatria básica: Pediatria Clínica Especializada. 9. ed. São Paulo: Sarvier, 2007. Tomo III. Cap. 8, p. 207-215.

5. Ministério da Saúde. Disponível em: $<\mathrm{http} / / /$ tabnet.datasus. gov.br/cgi/tabcgi.exe?sim/cnv/ obtrj.def $>$. Acessado em: 13/ 07/ 2010.

6. NASCIMENTO-CARVALHO, C. M. e SOUZA-MARQUES, H. H. Recomendação da Sociedade Brasileira de Pediatria para antibioticoterapia em crianças e adolescentes com pneumonia comunitária. Rev Panam Salud Publica. v.15, n.6, p. 380387, 2004;

7. AMARAL, João Joaquim Freitas do e PAIXÃO, Antônio Carvalho da. AIDPI para o Ensino Médico: Manual de Apoio. $3^{\mathrm{a}} \mathrm{ed}$. Brasília: Organização Pan-Americana da Saúde, 2004.179p.

8. MANDEL, Lionel A. e WUNDERINK, Richard. Pneumonia. In: FAUCI, Anthony S. et al. Harrison - Medicina Interna. 17.ed. Rio de Janeiro: McGraw-Hill Interamericana do Brasil, 2008. cap. 251, p. 1619-1628.

Endereço para Correspondência:

Daniel Machado

macdan_1972@hotmail.com

Av. Puglisi, 81 - apto. 31

Centro - Guarujá-SP

CEP: $11.410-001$ 